\title{
Impact of high-sensitivity cardiac troponin I assays on patients presenting to an emergency department with suspected acute coronary syndrome
}

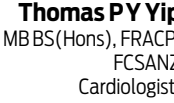

Heather M Pascoe MBBS(Hons), BMedSC Hospital Medical Officer

Stephen E Lane

$\mathrm{BSc}$ (Hons), PhD

Research Fellow

1 Geelong Cardiology Practice, Barwon Health, Geelong, VIC.

2 Biostatistics Unit Barwon Health, Geelong, VIC

thomasy@ barwonhealth.org.au

MJA 2014; 201: 158-161 doi: 10.5694/mjal3.00117

Editorial p 125 Research p 155
C ardiac troponins have long been used as the biomarker of choice to aid the diagnosis of acute myocardial infarction (AMI). ${ }^{1}$ In the consensus document on the universal definition of myocardial infarction, a key component in making the diagnosis of AMI is a rise and/or fall in levels of cardiac biomarkers, with at least one value above the 99th percentile value for a reference population. ${ }^{2}$ Recently, high-sensitivity cardiac troponin (hscTn) assays were introduced into clinical practice in Australia. These assays have improved detection at the lower limits of troponin levels, enabling troponin levels to be measured even in healthy individuals. ${ }^{3,4}$ They have been shown to detect myocardial infarction earlier than previously used assays. ${ }^{5-8}$ In addition, hscTn assays identify more patients with myocardial damage and those who are at an increased risk of future cardiac events. 9,10 However, with this increase in sensitivity comes a reduced specificity. ${ }^{6,11}$ Consequently, more patients may be labelled with a diagnosis of acute coronary syndrome (ACS). ${ }^{10}$ The importance of interpreting troponin results in the context of the clinical presentation and in association with complementary criteria has therefore been highlighted. 2,12,13

The aim of our study was to determine whether the introduction of hscTn-I assays had an impact on patients presenting with suspected ACS to the emergency department (ED) of a tertiary referral hospital, in terms of hospital admission rates, time spent in the ED and subsequent management and outcomes. We hypothesised that more patients would undergo coronary angiography and invasive treatment (percutaneous coronary intervention

Abstract

Objective: To determine whether introduction of high-sensitivity cardiac troponin I (hscTn-I) assays affected management of patients presenting with suspected acute coronary syndrome (ACS) to the emergency department (ED) of a tertiary referral hospital.

Design, patients and setting: $A$ retrospective analysis of all patients presenting to the Geelong Hospital ED with suspected ACS from 23 April 2010 to 22 April $2013-2$ years before and 1 year after the changeover to hscTn-I assays on 23 April 2012.

Main outcome measures: Hospital admission rates, time spent in the ED, rates of coronary angiography, rates of percutaneous coronary intervention $(\mathrm{PCl})$ and coronary artery bypass graft surgery (CABGS), rates of discharge with a diagnosis of ACS, and rates of inhospital mortality.

Results: 12360 consecutive patients presented with suspected ACS during the study period; 1897 were admitted to Geelong Hospital in the 2 years before and 944 in the 1 year after the changeover to hscTn-I assays. Comparing the two patient groups, there was no statistically significant difference in allhospital admission rates ( $95 \% \mathrm{Cl}$ for the difference, $-3.1 \%$ to $0.3 \% ; P=0.10$ ) or proportion of patients subsequently discharged with a diagnosis of ACS (95\% $\mathrm{Cl}$ for the difference, $-2.3 \%$ to $5.4 \% ; P=0.43$ ). After the changeover, the median time patients spent in the ED was $11.5 \%$ shorter $(3.85 \mathrm{~h} v 4.35 \mathrm{~h} ; 95 \% \mathrm{Cl}$ for the difference, -0.59 to $-0.43 ; P<0.001$ ) and the proportion of admitted patients undergoing coronary angiography was higher (53.4\% v $45.2 \% ; 95 \% \mathrm{Cl}$ for the difference, 4.3 to 12.0 percentage points; $P<0.001$ ), but there was no statistically significant rise in the proportion of patients who had invasive treatment $(\mathrm{PCl}$ and/or CABGS) $(95 \% \mathrm{Cl}$ for the difference, $-0.4 \%$ to $6.3 \% ; P=0.08)$. Inhospital mortality rates from ACS did not change significantly $(95 \% \mathrm{Cl}$ for the difference, $-1.5 \%$ to $0.8 \% ; P=0.43$ ).

Conclusion: The introduction of hscTn-I assays appeared to be associated with more rapid diagnosis, resulting in less time spent in the ED, without a change in hospital admission rates. A higher proportion of patients had coronary angiographies after the changeover, but there was no significant change in rates of invasive treatment or inhospital mortality.

$[\mathrm{PCI}]$ and/or coronary artery bypass graft surgery [CABGS]) after the changeover, owing to small increases in troponin levels that would previously have been below the diagnostic threshold.

\section{Methods}

Geelong Hospital is a major public teaching hospital in Victoria, Australia. It is part of Barwon Health and provides services to the Barwon region, which has a population of about
300000 . Geelong Hospital's ED is the only tertiary ED in Victoria that is located outside metropolitan Melbourne.

On 23 April 2012, hscTn-I assays were introduced to Geelong Hospital, with complete cessation of the previously used assay. The previously used assay is a troponin I assay that is carried out using the Dimension RxL Max analyser (Siemens Healthcare). It has a lower limit of detection (LoD) of $0.04 \mu \mathrm{g} / \mathrm{L}$, a 99th percentile of $0.07 \mu \mathrm{g} / \mathrm{L}$ with an imprecision of $15 \%-22 \%$ coefficient of variation $(\mathrm{CV})$, and a $\mathrm{CV}$ 
of $10 \%$ at $0.14 \mu \mathrm{g} / \mathrm{L}$; the diagnostic threshold is set at $0.10 \mu \mathrm{g} / \mathrm{L}$. The new assay is a hscTn-I assay that is carried out using the Dimension Vista system (Siemens Healthcare), which uses luminescent oxygen channelling immunoassay technology. It has an LoD of $0.015 \mu \mathrm{g} / \mathrm{L}$, a 99th percentile of $0.045 \mu \mathrm{g} / \mathrm{L}$, and a CV of $10 \%$ at $<0.04 \mu \mathrm{g} / \mathrm{L}$; the diagnostic threshold is set at $0.045 \mu \mathrm{g} / \mathrm{L}$. The hscTn-I assay is used, and its results interpreted, according to the 2011 addendum to the 2006 guidelines for management of ACS. ${ }^{14}$ In particular, the results were considered positive when the hscTn-I level was $\geqslant 99$ th percentile and there was a change of $\geqslant 50 \%$ above a baseline level.

The design of our study was a prepost changeover comparison. All patients who presented to the ED with suspected ACS from 23 April 2010 to 22 April 2013 were included. Suspected ACS was based on the diagnosis made by ED medical staff. The discharge diagnosis of ACS (defined by myocardial infarction or angina) was made by hospital in-house medical staff. Patients were categorised as presenting during the 2 years before the changeover or during the 1 year after the changeover. The 2-year and 1-year periods of data collection allowed for possible seasonality.

Data for consecutive patients were obtained from medical records provided by Health Information Services at Barwon Health. We had full access to both ED and hospital datasets of the studied population.

The numbers of patients presenting to the ED with suspected ACS, and the rates of admission to hospitals (both public and private), were determined. For those admitted to Geelong Hospital, we determined the percentage who underwent coronary angiography, the percentage who had invasive treatment, the percentage who were discharged with a diagnosis of ACS, and the rate of inhospital mortality due to ACS.

The study was approved by Barwon Health Human Research Ethics Committee.

\section{Statistical analysis}

The numbers of patients who presented to the ED with suspected ACS and the rates of hospital admission

1 Characteristics of patients who presented to the ED of Geelong Hospital with suspected ACS before and after changeover to hscTn-l assays $(N=12360)$

\begin{tabular}{|c|c|c|c|c|}
\hline & $\begin{array}{l}\text { Before changeover } \\
\quad(n=8090)\end{array}$ & $\begin{array}{l}\text { After changeover } \\
\qquad(n=4270)\end{array}$ & $\begin{array}{l}95 \% \mathrm{Cl} \text { for the } \\
\text { difference }\end{array}$ & $P$ \\
\hline Males & 4251 (52.5\%) & 2231 (52.2\%) & $-2.2 \%$ to $1.5 \%$ & 0.75 \\
\hline Age in years, median (IQR) & $59(27)$ & $59(26)$ & -1.0 to 1.0 & 0.68 \\
\hline Hypertension & 1088 (13.4\%) & $548(12.8 \%)$ & $-1.8 \%$ to $0.6 \%$ & 0.34 \\
\hline History of smoking & $2223(27.5 \%)$ & $1002(23.5 \%)$ & $-5.6 \%$ to $-2.4 \%$ & $<0.001$ \\
\hline Previous IHD & $1066(13.2 \%)$ & $525(12.3 \%)$ & $-2.1 \%$ to $0.4 \%$ & 0.16 \\
\hline
\end{tabular}

$\mathrm{ED}=$ emergency department. $\mathrm{ACS}=$ acute coronary syndrome. $\mathrm{hscTn}-\mathrm{I}$ = high-sensitivity cardiac troponin I. IQR = interquartile range. $\mathrm{IHD}=$ ischaemic heart disease.

2 Inhospital mortality due to ACS in patients admitted to Geelong Hospital after presenting to the ED with suspected ACS before and after changeover to hscTn-I assays*

Did not die from ACS in hospital

Before changeover $(n=1897)$

$1852(97.6 \%)$

$926(98.1 \%)$
Died from ACS in hospital

$45(2.4 \%)$

$18(1.9 \%)$

ACS = acute coronary syndrome. ED = emergency department. hscTn-I = high-sensitivity cardiac troponin I. * There was no statistically significant difference in inhospital mortality due to ACS between the pre-changeover and post-changeover periods $(P=0.43)$.

were assessed monthly to determine the presence of autocorrelation in the time series. There was no autocorrelation present, so standard linear models were used in a segmented regression analysis to investigate any shifts in level or changes in trend over time of the respective measures.

The rates of admission, coronary angiography, invasive treatment, discharge with ACS diagnosis and inhospital mortality due to ACS for the two patient groups were compared using 95\% confidence intervals, calculated by inverting the score test (all intervals are shown as post-changeover value minus pre-changeover value). Comparisons (and 95\% confidence intervals) for ordinal variables were calculated as the median of differences between patient groups. Comparisons for numerical variables (age and time spent in the ED) were calculated as difference in medians (and 95\% confidence intervals) using bootstrapping.

\section{Results}

During the study period, 12360 consecutive patients presented to the Geelong Hospital ED with suspected ACS; 1897 were admitted to Geelong Hospital before the changeover and 944 were admitted to Geelong Hospital after the changeover.

Characteristics of the two patient groups are shown in Box 1 . No statistically significant differences were found between the groups in terms of sex, age, hypertension and previous ischaemic heart disease. A lower prevalence of smoking history was noted in the post-changeover group, but no statistically significant difference in smoking history was noted among those admitted to Geelong Hospital (50.4\% [956/1897] before the changeover v 53.5\% [505/944] after the changeover; $95 \% \mathrm{CI}$ for the difference, - 7.0 to $0.8 ; P=0.12$ ). Data on diabetes were available but not analysed because of undercoding of this condition in medical records and changes in diabetes coding definitions during the study period.

\section{Presentation and admission rates}

There was no statistically significant difference over time in numbers of patients who presented to the ED with suspected ACS (mean difference, 18.8 patients/month; $95 \%$ CI for the difference, -3.2 to $40.7 ; P=0.09$ ). The all-hospital admission rates were not statistically different for the two patient groups: $31.1 \%$ (2518/8090) and $29.7 \%$ (1268/4270) for the prechangeover and post-changeover groups, respectively (95\% CI for the difference, $-3.1 \%$ to $0.3 \%$; $P=0.10$ ). The Geelong Hospital admission rates were also not statistically different: $23.4 \%(1897 / 8090)$ and $22.1 \%$ (944/4270), respectively (95\% CI for the difference, $-2.9 \%$ to $0.2 \% ; P=0.09$ ). 
Time in the emergency department

For patients presenting to the ED with suspected ACS, median time spent in the ED was $11.5 \%$ shorter in the post-changeover period compared with pre-changeover period $(3.85 \mathrm{~h} \mathrm{v}$ $4.35 \mathrm{~h} ; 95 \%$ CI for the difference, -0.59 to $-0.43 ; P<0.001)$.

\section{Discharge and management}

The proportion of ED patients with suspected ACS admitted to Geelong Hospital who were subsequently discharged with an ACS diagnosis was $56.5 \%(1072 / 1897)$ in the pre-changeover period and $58.1 \%$ (548/944) in the post-changeover period. This difference was not statistically different (95\% CI for the difference, $-2.3 \%$ to $5.4 \% ; P=0.43)$.

Among the patients admitted to Geelong Hospital, 45.2\% (858/1897) underwent coronary angiography in the pre-changeover period. This rose to $53.4 \%$ (504/944) following the introduction of hscTn-I assays, representing a statistically significant increase of 8.2 percentage points in absolute terms (95\% CI for the difference, 4.3 to 12.0 percentage points; $P<0.001)$. For invasive treatment (PCI and/or CABGS), proportions in the pre-changeover and post-changeover periods were $21.8 \%$ (413/1897) and $24.7 \%$ (233/944), respectively. Despite the slight increase, this difference was not statistically significant $(95 \%$ CI for the difference, $-0.4 \%$ to $6.3 \% ; P=0.08)$.

\section{Inhospital mortality}

The numbers of deaths from ACS that occurred in patients admitted to Geelong Hospital from the ED with suspected ACS are shown in Box 2. There was no statistically significant difference between the two periods (95\% CI for the difference, $-1.5 \%$ to $0.8 \% ; P=0.43$ ).

\section{Discussion}

In our study, introduction of hscTn-I assays did not change hospital admission rates for suspected ACS or the proportion of patients discharged with a diagnosis of ACS. The changeover appeared to be associated with more rapid diagnosis, resulting in patients spending less time in the ED. Although there was an increase in the proportion of patients for whom coronary angiography was performed, there was no significant increase in invasive treatment or change in inhospital mortality.

Some authors have reported that the number of patients labelled with ACS has increased after introduction of hscTn assays, owing to lowering of the diagnostic threshold. ${ }^{10,15}$ In particular, it has been suggested that adopting the 99th percentile value for plasma troponin (as recommended in the consensus document on the universal definition of myocardial infarction $^{2}$ ) would increase the number of people receiving a diagnosis of AMI by $47 \%$. 10 Our results indicate that such an increase in the diagnosis of ACS did not occur.

Our experience suggests that "ultraprecision" in the measurement of cardiac troponin may not cause further changes in the rate of clinical diagnosis of ACS. This is similar to findings from the Brigham and Women's Hospital clinical laboratories in 2007 - a doubling in the number of positive troponin results in the ED after introducing a hscTn-I assay, but no change in the frequency of a final diagnosis of ACS. ${ }^{15}$ This suggests that proper interpretation of troponin results (including rise and fall in troponin levels determined by hscTn assays ["delta troponin"]) with appropriate attention to clinical signs and symptoms, electrocardiography results, and other complementary data may prevent increases in rates of diagnosis of ACS that might otherwise be associated with use of hscTn assays.

Nonetheless, patients in our study spent less time in the ED after the changeover to hscTn-I assays. With earlier generation cardiac troponin assays, troponin rise can only be detected after a post-AMI period of at least 3-4 hours; also, repeat sampling 6-12 hours later is often needed to exclude the possibility of myocardial damage. ${ }^{16,17}$ With hscTn assays, a rise can be identified earlier and it can reduce the time to rule in or rule out a diagnosis of AMI. ${ }^{5-8,11,18-20}$ The cumulative sensitivity of a sensitive cardiac troponin I assay using a diagnostic threshold of 99 th percentile $(0.07 \mu \mathrm{g} / \mathrm{L})$ has been reported as $93 \%$ at $30 \mathrm{~min}-$ utes after presentation, $98 \%$ at 2 hours, and $100 \%$ at 3 hours, with a specificity of $57 \%$ at 2 hours and $54 \%$ at 3 hours. ${ }^{21}$
Delays in ruling out AMI contribute to overcrowding in EDs, which is associated with substantial costs. In 2011, it was suggested that initially undetectable levels of troponin based on hscTn$\mathrm{T}$ assays would have ruled out AMI in $27.7 \%$ of patients with a sensitivity of $100 \% .{ }^{19}$ In 2012 , an algorithm for using hscTn-T assays to rule in and rule out AMI was published; it incorporated baseline troponin thresholds and thresholds for rises in troponin during the first hour. ${ }^{20}$ Applied to 436 patients, within the first hour of presentation, AMI could be ruled out for $60 \%$ of patients and ruled in for $17 \%$, leaving 23\% in an "observation zone". No AMI was missed in the rule-out group. However, no such algorithm was used in our study population.

The impact that hscTn assays have had on early diagnosis of AMI5-8,11,19,20 and survival prognostication 9,22 has been reported extensively. Although it has been reported that early generation "sensitive" troponin assays capable of lowering the diagnostic threshold (but not to the level of the 99th percentile) were associated with a change in clinical practice (more referrals to cardiac specialists, more coronary angiographies and wider use of evidenced-based treatments), ${ }^{23}$ no previous studies have directly examined the effect of the introduction of hscTn assays (now with a diagnostic threshold down to the 99th percentile) on coronary angiography and invasive treatment rates. As hypothesised, we observed a significant increase in coronary angiography rates. This could not be explained by any change in cardiac catheterisation laboratory availability as there had been no significant structural change during the study period.

However, we did not observe a proportional increase in invasive treatment rates, which remained stable. During the study period, there was no change in funding practice that might have affected the way PCI and CABGS services were delivered. Understandably, patients not receiving invasive treatment may have been started on medical therapy or may have had existing medications up-titrated (factors that we did not examine). Some of these patients may have had troponin rises due to type 2 myocardial infarction (eg, sepsis, hypertensive crisis or tachycardic atrial 
fibrillation 2), or other pathophysiological situations leading to myocardial cell death.8,24 With even more sensitive cardiac troponin assays ("ultrasensitive" or "novel highly sensitive" assays) in the pipeline, $12,13,25$ these new generations of biomarker assays will continue to present myriad challenges for clinicians evaluating patients with suspected ACS. The adoption of this quickly evolving technology should be accompanied by algorithms — which, ideally, should be validated - to enhance assay performance in terms of specificity, positive predictive values and ability to guide referrals, additional investigations and treatment.

Our study had six main limitations. First, it was retrospective and we relied on diagnoses given by ED staff to determine which patients presented with suspected ACS. Second, our findings represent one tertiary centre. However, given the diverse population and large area that the Geelong Hospital ED serves, our study population is likely to be representative of the general Australian population presenting with suspected ACS. Third, we noted a non-significant trend towards more invasive treatment following the introduction of hscTn-I assays, so further studies might show a significant increase. Fourth, we assumed that any changes in the studied parameters were only related to the introduction of hscTn-I assays, and not any concomitant external factors. The overall baseline characteristics of the patients in the pre-changeover and post-changeover periods do, however, match quite well; the only significant difference was in smoking history, which probably had a minimal effect on admission rates and time spent in the ED and no effect on the other studied parameters, which involved inpatient management and outcomes. During the study period, there were no other new cardiac tests introduced, and no major changes in hospital management or the size and number of operating units in the ED. The National Emergency Access Target (NEAT) initiative, which encourages shorter ED stay, was in place before the start of the study period. This initiative involved incremental targets for improving efficiency over the study period, so it is hard to know whether shorter
ED stay in our study population was a result of hscTn-I assays or the NEAT initiative; or better still, whether use of hscTn-I assays helped in the NEAT initiative. Fifth, we evaluated one hscTn assay. We hypothesise that our findings can be generalised to other cardiac troponin assays that have similar sensitivity and precision. Finally, the impact of introducing hscTn-I assays encompasses the performance of the biomarker itself and the preparedness of clinicians in interpreting the results.

In conclusion, the introduction of hscTn-I assays was associated with shorter ED stays and an increase in coronary angiography rates but no increase in hospital admission rates, invasive treatment rates or inhospital mortality. These findings are reassuring and indicate that concerns about a flood of coronary admissions and interventions as a result of the higher-sensitivity assays are ill founded. Analysis of ongoing clinical experience with these assays is likely to lead to improvement in patient management through refinement of management algorithms.

Acknowledgements: We thank the staff of the Information Technology Department and the Decision Support Unit (Health Information Services) of Barwon Health for collating the dataset, particularly Brodie de Munk for her exceptional efforts.

Competing interests: No relevant disclosures.

Received 24 Nov 2013, accepted 10 Apr 2014.

1 Morrow DA, Cannon CP, Jesse RL, et al. National Academy of Clinical Biochemistry Laboratory Medicine Practice Guidelines: clinical characteristics and utilization of biochemical markers in acute coronary syndromes. Circulation 2007; 115: e356-e375.

2 Thygesen K, Alpert JS, Jaffe AS, et al. Third universal definition of myocardial infarction. J Am Coll Cardiol 2012; 60: 1581-1598.

3 White HD. Higher sensitivity troponin levels in the community: what do they mean and how will the diagnosis of myocardial infarction be made? Am Heart J 2010; 159: 933-936.

4 Zethelius B, Johnston N, Venge P. Troponin I as a predictor of coronary heart disease and mortality in 70-year-old men: a communitybased cohort study. Circulation 2006; 113: 1071-1078.

5 Reiter M, Twerenbold R, Reichlin T, et al. Early diagnosis of acute myocardial infarction in the elderly using more sensitive cardiac troponin assays. Eur Heart J 2011; 32: 1379-1389.

6 Keller T, Zeller T, Peetz D, et al. Sensitive troponin I assay in early diagnosis of acute myocardial infarction. N Engl J Med 2009; 361: 868-877.

7 Reiter M, Twerenbold R, Reichlin T, et al. Early diagnosis of acute myocardial infarction in patients with pre-existing coronary artery disease using more sensitive cardiac troponin assays. Eur Heart J 2012; 33: 988-997.
8 Bonaca MP, Ruff CT, Kosowsky J, et al. Evaluation of the diagnostic performance of current and next-generation assays for cardiac troponin I in the BWH-TIMI ED Chest Pain Study. Eur Heart J Acute Cardiovasc Care 2013; 2: 195-202.

9 Ndrepepa G, Braun S, Mehilli J, et al. Prognostic value of sensitive troponin $T$ in patients with stable and unstable angina and undetectable conventional troponin. Am Heart J 2011; 161 : 68-75.

10 Mills NL, Lee KK, McAllister DA, et al. Implications of lowering threshold of plasma troponin concentration in diagnosis of myocardial infarction: cohort study. BMJ 2012; 344: el533.

11 Reichlin T, Hochholzer W, Bassetti S, et al. Early diagnosis of myocardial infarction with sensitive cardiac troponin assays. NEngl J Med 2009; 361: 858-867.

12 Thygesen K, Mair J, Katus H, et al. Recommendations for the use of cardiac troponin measurement in acute cardiac care. Eur Heart J 2010; 31: 2197-2204.

13 Twerenbold R, Jaffe A, Reichlin T, et al. Highsensitive troponin T measurements: what do we gain and what are the challenges? Eur Heart J 2012; 33: 579-586.

14 Chew DP, Aroney CN, Aylward PE, et al. 2011 addendum to the National Heart Foundation of Australia/Cardiac Society of Australia and New Zealand guidelines for the management of acute coronary syndromes (ACS) 2006. Heart Lung Circ 2011; 20: 487-502.

15 Melanson SE, Conrad MJ, Mosammaparast $\mathrm{N}$, Jarolim P. Implementation of a highly sensitive cardiac troponin I assay: test volumes, positivity rates and interpretation of results. Clin Chim Acta 2008; 395: 57-61.

16 Bassand J, Hamm CW, Ardissino D, et al. Guidelines for the diagnosis and treatment of non-ST-segment elevation acute coronary syndromes. Eur Heart J 2007; 28: 1598-1660.

17 Anderson JL, Adams CD, Antman EM, et al. ACC/AHA 2007 guidelines for the management of patients with unstable angina/non-STelevation myocardial infarction. J Am Coll Cardiol 2007; 50: el-el57.

18 Thygesen K, Mair J, Giannitsis E, et al. How to use high-sensitivity cardiac troponins in acute cardiac care. Eur Heart J 2012; 33: 2252-2257.

19 Body R, Carley S, McDowell G, et al. Rapid exclusion of acute myocardial infarction in patients with undetectable troponin using a high-sensitivity assay. J Am Coll Cardiol 2011; 58: 1332-1339.

20 Reichlin T, Schindler C, Drexler B, et al. Onehour rule-out and rule-in of acute myocardial infarction using high-sensitivity cardiac troponin T. Arch Intern Med 2012; 172: 1211-1218.

21 Eggers KM, Oldgren J, Nordenskjöld A, Lindahl B. Diagnostic value of serial measurement of cardiac markers in patients with chest pain: limited value of adding myoglobin to troponin I for exclusion of myocardial infarction. Am Heart J 2004; 148: 574-581

22 Omland T, de Lemos JA, Sabatine MS, et al. A sensitive cardiac troponin T assay in stable coronary artery disease. N Engl J Med 2009; 361: 2538-2547.

23 Baver A, Gawaz M. Sensitive cardiac troponin assays [letter]. N Engl J Med 2009; 361: 2575-2577.

24 Agewall S, Giannitsis E, Jernberg T, Katus $\mathrm{H}$. Troponin elevation in coronary vs. noncoronary disease. Eur Heart J 2011; 32: 404-411.

25 de Lemos JA. Increasingly sensitive assays for cardiac troponins: a review. JAMA 2013; 309: 2262-2269. 